\title{
Évaluation de l'érosion hydrique dans des bassins versants de la zone semi-aride tunisienne avec les modèles RUSLE et MUSLE couplés à un Système d'information géographique
}

\author{
Khaoula Khemiri ${ }^{1,2, *}$ et Sihem Jebari ${ }^{1}$ \\ ${ }^{1}$ Institut national de la recherche en génie rural, eaux et forêts, Ariana, Tunisie \\ 2 École nationale d'ingénieurs de Tunis, Le Belvédère-Tunis, Tunisie
}

\begin{abstract}
Résumé - L'érosion hydrique est une préoccupation agronomique, économique et environnementale importante en milieu semi-aride méditerranéen. Le choix de la méthode pour l'estimer dépend des données disponibles et des conditions de la zone d'étude. Cet article vise à quantifier et spatialiser l'érosion hydrique en utilisant l'équation universelle des pertes en sol (Universal Soil Loss Equation [USLE]) dans ses versions modifiée (Modified - MUSLE) et révisée (Revised - RUSLE) et couplées à un Système d'information géographique (SIG) : MUSLE-SIG et RUSLE-SIG. Les données de terrain de deux bassins versants situés au centre de la Tunisie, Fidh Ali et Fidh Ben Naceur, ont été utilisées. Les deux modèles ont été calés et validés pour la période 1994-2000. Les résultats montrent une simulation satisfaisante de la réalité en termes de dégradation du paysage, avec des résultats assez similaires pour les deux modèles. RUSLE-SIG, plus récent, est largement utilisé par les chercheurs en Tunisie, mais la version calibrée de MUSLE-SIG donne ici d'aussi bons résultats. Ces deux modèles pourraient donc être utilisés par les organismes de développement agricole et de lutte contre l'érosion dans les zones semi-arides de Tunisie, le choix de l'un ou de l'autre étant tributaire de la disponibilité des données nécessaires.
\end{abstract}

Mots clés : érosion hydrique / RUSLE / MUSLE / SIG / aménagement anti-érosif

\begin{abstract}
Water erosion assessment in watersheds of the Tunisian semi-arid area with RUSLE and MUSLE models coupled with a Geographic Information System. Water erosion is an important agronomic, economic and environmental concern in the semi-arid Mediterranean environment. Characterization of the process and choice of a method for estimating it depends on available data and characteristics of the study area. This article aims at quantifying and spatializing water erosion using the Modified (MUSLE) and Revised (RUSLE) Universal Soil Loss Equation coupled with a Geographic Information System (GIS): MUSLE-SIG and RUSLE-SIG. Erosion was modeled in two watersheds located in central Tunisia, Fidh Ali and Fidh Ben Naceur. Field data from 1994 to 2000 were used to calibrate RUSLE-GIS and MUSLE-GIS. Results showed a faithful modeling of reality in terms of landscape degradation. Both models gave relatively similar results. While RUSLE-GIS, more recent, is widely used by researchers in Tunisia, the calibrated version of MUSLE-GIS also gave satisfaction in simulating water erosion. Consequently, both models could be used in Tunisia by development agencies aiming at reducing soil erosion, the choice of one or the other depending on the available data.
\end{abstract}

Keywords: water erosion / RUSLE / MUSLE / GIS / anti-erosion devices

\section{Introduction}

L'érosion hydrique est un problème environnemental mondial, entravant gravement le développement durable (Gao et Wang, 2018). Ce phénomène résulte d'un ensemble

\footnotetext{
*Auteur de correspondance : khemiri.khaoula@gmail.com
}

de processus complexes et interdépendants, liés à la pluie, au ruissellement, à la topographie, à la texture du sol, à la couverture végétale, aux techniques culturales et aux éventuels aménagements. Tous ces facteurs réunis contribuent directement ou indirectement au détachement et au transport des particules du sol (Wischmeier et Smith, 1965). En milieu semiaride méditerranéen, l'érosion est très active et préjudiciable. Elle est particulièrement importante en Tunisie (CNEA, 2008) 
et n'a cessé de s'y accentuer, du fait des conditions climatiques, physiques, géomorphologiques, agricoles, pastorales et socio-économiques du pays. L'érosion hydrique provoque non seulement la dégradation du paysage agricole, mais aussi l'envasement des infrastructures hydro-agricoles. La modélisation peut être un outil d'aide à la décision et à la gestion efficiente et durable des ressources en eau et en sol si les modèles sont adaptés aux conditions locales.

Divers chercheurs ont étudié l'érosion en se basant sur des méthodes empiriques, statistiques ou physiques (e.g., Wischmeier et Smith, 1965; Le Bissonnais et al., 2002 ; Kirkby et al., 2003). Le choix d'une méthode dépend aussi bien de l'objectif visé que de la pertinence de cette méthode dans les conditions de la zone d'étude et de la faisabilité de son utilisation compte tenu des données disponibles. L'équation universelle des pertes en sol USLE (Universal Soil Loss Equation) a été élaborée par Wischmeier et Smith (1965) aux États-Unis. Sa version modifiée MUSLE (Modified Universal Soil Loss Equation) a été mise au point par Williams (1975) et une version révisée RUSLE (Revised Universal Soil Loss Equation) a ensuite été produite par Renard et al. (1997).

Des études menées partout dans le monde continuent à améliorer le paramétrage et l'application des modèles MUSLE et RUSLE dans différents contextes (Ketema et Dwarakish, 2019). Notre travail vise à contribuer à ces efforts pour caler, adapter, valider et assurer l'adoption de ces modèles « universels» dans des conditions climatiques spécifiques et dans un contexte de recherche et de développement nécessitant beaucoup d'efforts pour mettre en œuvre ces méthodes. La modélisation peut permettre une meilleure gestion des ressources naturelles et un aménagement du paysage agricole mieux ciblé et moins coûteux. Bien que le modèle original USLE ait été mis au point sur des petites parcelles, son utilisation à des échelles plus larges, comme les bassins versants, pourrait fournir des estimations intéressantes des pertes en sol, à condition que l'hypothèse de l'homogénéité et de la conformité de l'échelle utilisée pour l'estimation des facteurs de la modélisation soit vérifiée. Pour cela, la zone d'étude doit être discrétisée et décomposée en une série de zones ayant une utilisation et un traitement des terres relativement homogènes (Wischmeier et Smith, 1965; Smith et al., 2000). Plusieurs études ont ainsi confirmé que lorsque les paramètres (climat, pluviométrie, couverture végétale, texture des sols) des modèles RUSLE et MUSLE sont soigneusement adaptés à la zone d'étude, l'incertitude de ces modèles n'est pas plus grande ailleurs dans le monde qu'aux États-Unis. Leurs performances pour l'estimation des pertes en sol sont ainsi jugées assez bonnes d'un point de vue scientifique (Kinnell, 2010; Yue et al., 2016; Alewell et al., 2019). En Tunisie, l'usage de l'équation universelle des pertes en sol (USLE) a été validé par Masson (1970).

Le présent travail se base sur les modèles MUSLE et RUSLE couplés à un Système d'information géographique (SIG). Cette approche rend possible l'estimation de l'érosion hydrique et de sa distribution spatiale dans des zones vastes, à l'échelle du bassin versant et du pays, voire du continent (Smith et al., 2000; Renard et al., 2011; Jebari et al., 2012; Borrelli et al., 2017).

Cette étude vient continuer les travaux entrepris de longue date en Tunisie, comme ceux du CREGR (1965), de Guy (1967), Masson (1970), Heush (1982), El Amami (1984), du
PNUD/FAO (1991), de Mhiri et al. (1994), Saadaoui (1996), Nasri (2002) et Jebari (2009). En accord avec les suggestions de Smith et al. (2000), Sun et al. (2014), Jebari et al. (2015) et Borrelli et al. (2017), elle étend l'usage scientifique de ces approches à différents contextes, afin de pouvoir, dans le futur, les transmettre aux praticiens du développement agricole et de la lutte anti-érosive des pays du Sud.

\section{Matériel et méthodes}

\subsection{Site d'étude}

La zone d'étude est localisée dans la partie nord-est de la délégation de Haffouz, dans le gouvernorat de Kairouan, au centre de la Tunisie. Elle est constituée de deux bassins versants, Fidh Ali et Fidh Ben Naceur, situés respectivement à 330 et $440 \mathrm{~m}$ d'altitude (Fig. 1). La zone d'étude fait partie de la Dorsale tunisienne et a un climat semi-aride. Les précipitations moyennes annuelles dans les deux bassins versants sont de $292 \mathrm{~mm}$ pour Fidh Ali et $237 \mathrm{~mm}$ pour Fidh Ben Naceur (Jebari, 2009). Ces deux bassins couvrent, respectivement, une superficie de 238 ha et 169 ha. Les cours d'eaux principaux sont très encaissés dans un substratum marneux et viennent alimenter l'oued Merguellil, $20 \mathrm{~km}$ en amont du barrage El Haouareb. Deux lacs collinaires à fins agricoles (irrigation) ont été créés à l'exutoire de ces bassins au début des années 1990, avec des capacités initiales de 134 $700 \mathrm{~m}^{3}$ (Fidh Ali) et $47110 \mathrm{~m}^{3}$ (Fidh Ben Naceur)

Les réservoirs collinaires de Fidh Ali et de Fidh Ben Naceur ont fait l'objet de trois campagnes de mesures bathymétriques pour le premier $(1996,1998,1999)$ et de quatre pour le second $(1996,1997,1998,1999)$. L'envasement moyen annuel est de $22,7 \mathrm{tha}^{-1} \mathrm{an}^{-1}$ pour Fidh Ali et $14,5 \mathrm{tha}^{-1} \mathrm{an}^{-1}$ pour Fidh Ben Naceur (Jebari et al., 2010). Ces valeurs sont très supérieures aux pertes en sol tolérables, estimée par Masson (1972) à 2,5, 5 et $10 \mathrm{tha}^{-1} \mathrm{an}^{-1}$ pour des sols minces, moyens et épais. Ces deux bassins versants présentent donc des zones très vulnérables à l'érosion.

Durant la période d'étude (1994-2000), la plupart des évènements pluvieux ont généré du ruissellement (Tab. 1a et $1 \mathrm{~b}$ ), avec des processus érosifs en nappe, rigole et ravine (Toy et al., 2002; Jebari, 2009). Cette sensibilité à la dégradation s'explique par des alternances calcaire/marne donnant des sols limono-argileux à argiles actives qui génèrent de l'érosion en rigoles et en ravines (Collinet et al., 2002).

\subsection{Données utilisées}

Le modèle numérique de terrain (MNT) est dérivé des données Shuttle Radar Topography Mission (SRTM). Elles ont une résolution de $30 \mathrm{~m} \times 30 \mathrm{~m}$ et sont accessibles sur le site USGS (https://earthexplorer.usgs.gov/; accès le 30/11/ 2019). La carte pédologique, la carte d'occupation des sols et la carte des aménagements de conservation des eaux et des sols (CES) sont issues de la carte agricole du gouvernorat de Kairouan (ministère de l'Agriculture et des Ressources hydrauliques, mission 1998, échelle 1/20 000). Les photos aériennes utilisées pour actualiser et valider les cartes d'occupation des sols et des aménagements CES proviennent de Google Earth et datent de 1998. 


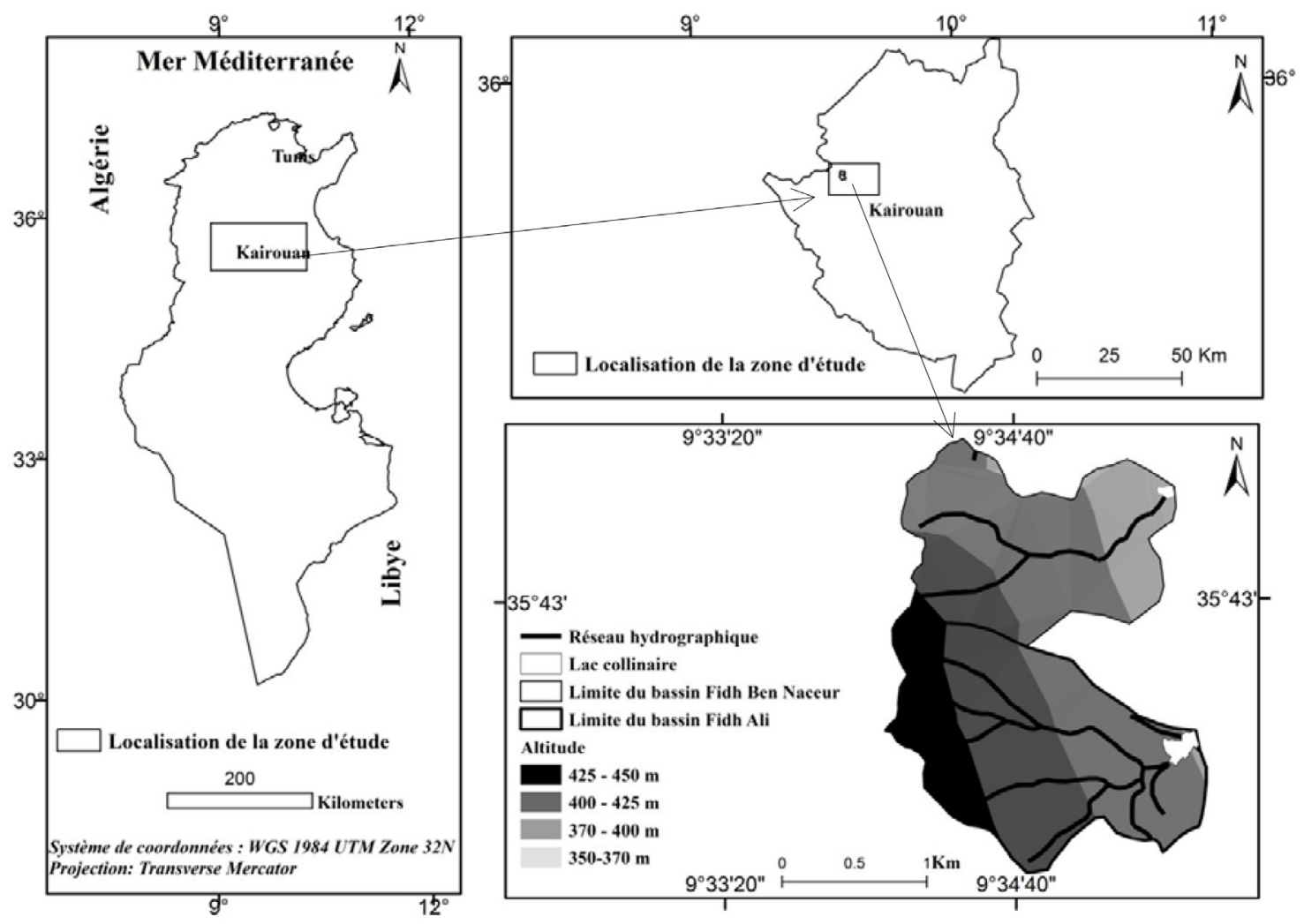

Fig. 1. Localisation et topographie des bassins versants de Fidh Ali et Fidh Ben Naceur.

Fig. 1. Location of the study area and topography of the watersheds.

Tableau 1a. Caractéristiques des crues maximales pour les bassins versants Fidh Ali et Fidh Ben Naceur (HYDROMED-DCES/IRD, 1994-2002). Table. Characteristics of maximum floods for the Fidh Ali and Fidh Ben Naceur watersheds (HYDROMED-DCES/IRD, 1994-2002).

\begin{tabular}{lllll}
\hline Bassin versant & Capacité initiale $\left(\mathrm{m}^{3}\right)$ & Date de la crue & Volume de la crue $\left(\mathrm{m}^{3}\right)$ & $Q_{\text {max }}\left(\mathrm{m}^{3} \mathrm{~s}^{-1}\right)$ \\
\hline Fidh Ali & 134700 & $03 / 10 / 1994$ & 80200 & 37,1 \\
Fidh Ben Naceur & 47110 & $17 / 09 / 1997$ & 23822 & 27,9 \\
\hline
\end{tabular}

Tableau 1b. Caractérisation des évènements pluvieux d'une durée de 15 min pour les bassins versants Fidh Ali et Fidh Ben Naceur (Jebari et al., 2008). Table. Characterization of rainy events lasting 15 min for the Fidh Ali and Fidh Ben Naceur watersheds (Jebari et al., 2008).

\begin{tabular}{lllll}
\hline Bassin versant & $\begin{array}{l}\text { Intensité moyenne de } \\
\text { l'évènement }(\mathrm{mm})\end{array}$ & Durée moyenne $(\mathrm{min})$ & $I_{15}$ moyenne $\left(\mathrm{mm} \mathrm{h}^{-1}\right)$ & ${\text { Écart-type } I_{15}\left(\mathrm{~mm} \mathrm{~h}^{-1}\right)}$ \\
\hline Fidh Ali & 22,3 & 87,6 & 53,1 & 16,9 \\
Fidh Ben Naceur & 21,5 & 99,0 & 53,1 & 13,9 \\
\hline
\end{tabular}

Les données pluviométriques et hydrométriques sont issues des observations effectuées dans le cadre du réseau pilote de surveillance des lacs collinaires. Elles ont été publiées dans les annuaires hydrologiques (DCES/IRD, 1994-2001) du programme HYDROMED (Réseau d'observation hydrologique). Les données sont mesurées par un pluviomètre journalier, un pluviographe, et un limnigraphe. Le pluviographe permet d'enregistrer les hauteurs de pluies sur des intervalles de temps très courts et de restituer les hyéto- grammes (courbes d'intensité) des averses. Le limnigraphe permet d'enregistrer les hauteurs du plan d'eau de la retenue et d'avoir les hydrogrammes des crues, en particulier pendant les phases de déversement (Camus et al., 1995). Chaque lac est équipé de deux centrales d'acquisition automatique de données. La première est reliée à un capteur pluviométrique à augets basculeurs (précision : 0,5 $\mathrm{mm}$ de pluie) et la seconde à une sonde immergée mesurant le niveau de l'eau au cm près (Boufaroua et al., 2000). L'évacuateur de crue est aménagé 


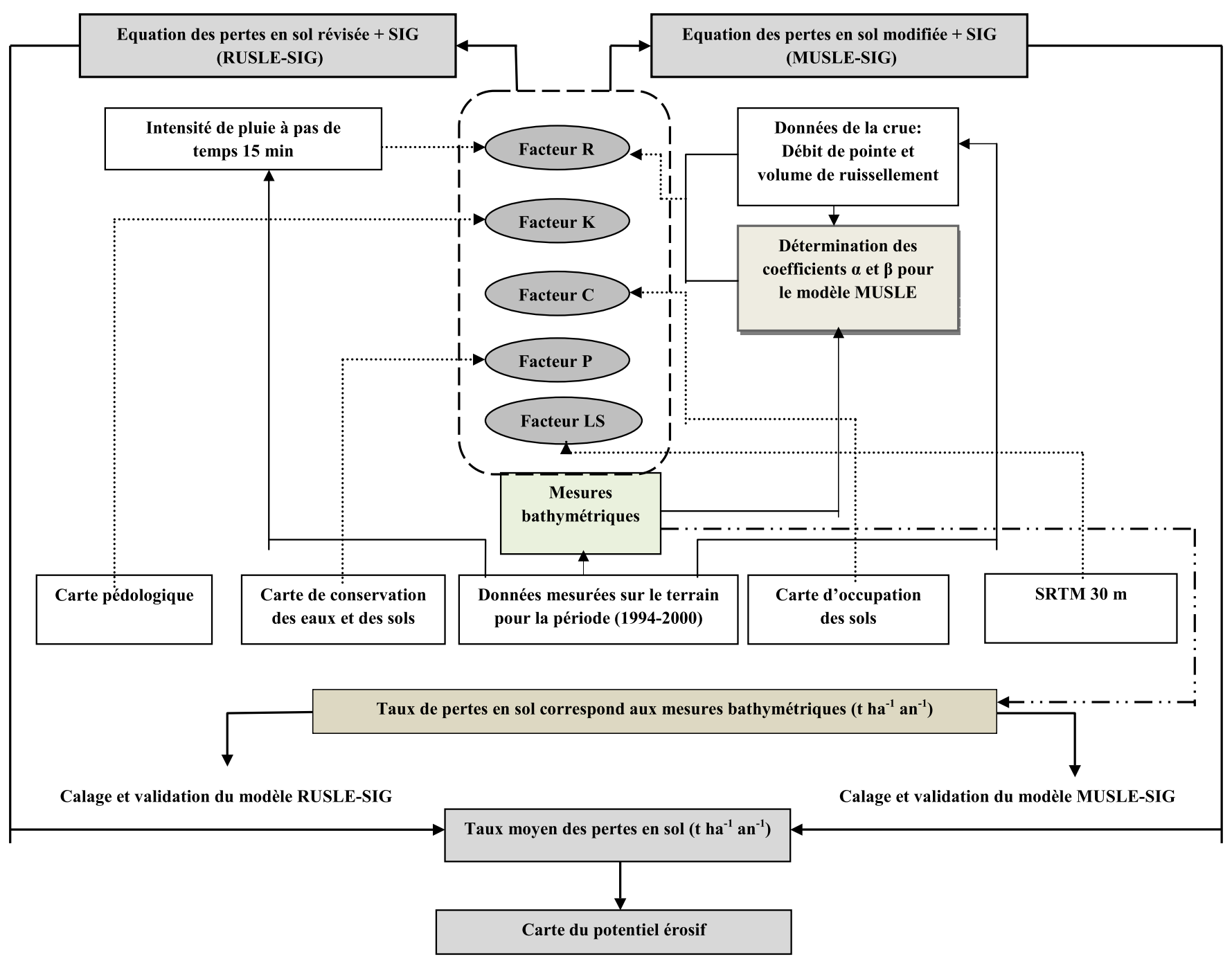

Fig. 2. Schéma descriptif de la méthodologie adoptée.

Fig. 2. Diagram of the methodological steps.

avec un seuil déversant permettant d'estimer les débits. Les données sont consignées dans une banque de données géoréférencées, avec moins de $1 \%$ de données manquantes.

Les mesures d'envasement sont réalisées à partir des sondages $\mathrm{du}$ fond vaseux. Entre 500 et 1000 points sont mesurés suivant des transversales réparties sur l'ensemble du lac. La bathymétrie est calculée par des méthodes géostatistiques (Camus et al., 1995). La perte de volume entre deux mesures est considérée égale au volume de sédiments transportés diminué de celui qui a quitté la retenue par déversement (Onibon et al., 1997 ; Albergel et al., 2004).

\subsection{Modèles des pertes en sol}

Il existe de nombreux modèles pour estimer l'érosion hydrique à différentes échelles spatio-temporelles. Le modèle USLE (Wischmeier et Smith, 1965) avec ses deux versions MUSLE (Williams, 1975) et RUSLE (Renard et al., 1997) est largement utilisé à travers le monde. Plusieurs études ont été effectuées pour déterminer ses paramètres en Tunisie (CREGR, 1965 ; Masson, 1970).

\subsection{1 Équation des pertes en sol modifiée (MUSLE)}

Le modèle MUSLE (Williams, 1975) est la version modifiée du modèle USLE qui évalue l'érosion hydrique pendant la crue $A_{\text {MUSLE }}$ (eq. (1)) en fonction de cinq facteurs d'érosion (Fig. 2) (Cinnirella et al., 1998) :

$$
\mathrm{A}_{\text {MUSLE }}=\alpha\left(\mathrm{Qq}_{\mathrm{p}}\right)^{\beta} \times \mathrm{K} \times \mathrm{LS} \times \mathrm{C} \times \mathrm{P},
$$

$\alpha$ et $\beta$ sont deux paramètres du modèle MUSLE. $Q$ est le volume du ruissellement $\left(\mathrm{m}^{3}\right) . \mathrm{q}_{\mathrm{p}}$ est le débit de pointe $\left(\mathrm{m}^{3} \mathrm{~s}^{-1}\right)$ pendant la crue. $\mathrm{K}$ est le facteur d'érodibilité du sol $\left(\mathrm{th} \mathrm{MJ} \mathrm{mm}^{-1}\right)$. LS est le facteur topographique (sans unité). $\mathrm{C}$ est le facteur de gestion de la couverture végétale (sans unité). $\mathrm{P}$ est le facteur d'aménagement anti-érosif (sans unité).

\subsection{2 Équation des pertes en sol révisée (RUSLE)}

Le modèle RUSLE (Renard et al., 1997) est la version révisée du modèle USLE. Il possède de nombreuses modifications pour l'estimation de l'érosion hydrique en 
tenant compte du ruissellement et en introduisant un nouveau terme d'érosivité pluie-ruissellement (Renard et al., 2011). RUSLE prévoit la perte en sol moyenne annuelle $A_{\text {RUSLE }}$ $\left(\mathrm{tha}^{-1} \mathrm{an}^{-1}\right)$ (eq. (2)) en fonction de cinq facteurs d'érosion (Fig. 2) :

$$
\mathrm{A}_{\mathrm{RUSLE}}=\mathrm{R}_{\mathrm{RUSLE}} \times \mathrm{K} \times \mathrm{LS} \times \mathrm{C} \times \mathrm{P},
$$

$\mathrm{R}_{\mathrm{RUSLE}}$ est le facteur d'érosivité de pluie-ruissellement (MJ $\mathrm{mm} \mathrm{ha}{ }^{-1} \mathrm{~h}^{-1} \mathrm{an}^{-1}$ ) pour le modèle RUSLE. $\mathrm{K}, \mathrm{LS}, \mathrm{C}$ et $\mathrm{P}$ sont les mêmes facteurs que dans le modèle MUSLE.

\subsection{Description des facteurs du modèle}

\subsubsection{Facteur d'érosivité du ruissellement $R_{\text {MUSLE }}$ pour le} modèle MUSLE

Le facteur $\mathrm{R}_{\text {MUSLE }}$ (eq. (3)) se calcule en fonction du débit de pointe et du volume de ruissellement à l'échelle de la crue (Cinnirella et al., 1998):

$$
\mathrm{R}_{\text {MUSLE }}=\sum_{\mathrm{j}=1}^{n} \frac{\alpha}{\mathrm{A}_{\mathrm{w}}}\left(\mathrm{q}_{\mathrm{p}, \mathrm{j}} \mathrm{Q}_{\mathrm{j}}\right)^{\beta}
$$

où $n$ est le nombre total des crues durant l'année. $\alpha$ et $\beta$ sont deux paramètres du modèle MUSLE. $Q_{j}$ est le volume du ruissellement pendant la crue $\mathrm{j}\left(\mathrm{m}^{3} \mathrm{~s}^{-1}\right)$. $\mathrm{q}_{\mathrm{p}, \mathrm{j}}$ est le débit de pointe pendant la crue $\mathrm{j}\left(\mathrm{m}^{3}\right) . \mathrm{A}_{\mathrm{w}}$ est la superficie de la zone d'étude (ha).

Dans le présent travail, toutes les crues observées entre deux mesures bathymétriques pendant la période d'étude (1994-2000) sont prises en compte, et reconstituées suivant un modèle de bilan hydrologique au pas de temps de 5 minutes.

Sur un intervalle de temps $\mathrm{t}$, à partir de l'équation de la conservation des volumes, l'équation générale du bilan hydrique d'une retenue et les débits instantanés de la crue entrant dans la retenue sont calculés par les équations (4) et (5) (DCES/IRD, 1994-2001) :

$$
\begin{aligned}
\Delta \mathrm{V} & =\left(\mathrm{V}_{\mathrm{r}}+\mathrm{V}_{\mathrm{ecs}}+\mathrm{V}_{\mathrm{p}}+\mathrm{V}_{\mathrm{f}}\right) \\
& -\left(\mathrm{V}_{\mathrm{ev}}+\mathrm{V}_{\mathrm{d}}+\mathrm{V}_{\vartheta \mathrm{i}}+\mathrm{V}_{\mathrm{i}}+\mathrm{V}_{\mathrm{pr}}\right)
\end{aligned}
$$

où $\Delta \mathrm{V}$ est la variation de stock dans la retenue $\left(\mathrm{m}^{3}\right) . \mathrm{V}_{\mathrm{r}}$ les apports en provenance du ruissellement des versants $\left(\mathrm{m}^{3}\right)$. $\mathrm{V}_{\mathrm{ecs}}$ représente les apports souterrains $\left(\mathrm{m}^{3}\right) . \mathrm{V}_{\mathrm{p}}$ représente les apports des précipitations tombant directement sur la retenue $\left(\mathrm{m}^{3}\right) . \mathrm{V}_{\mathrm{f}}$ représente les apports dus à la fonte des neiges $\left(\mathrm{m}^{3}\right)$. $\mathrm{V}_{\mathrm{ev}}$ est le volume d'eau évaporé $\left(\mathrm{m}^{3}\right) . \mathrm{V}_{\mathrm{d}}$ est le volume d'eau sortant de la retenue par déversement $\left(\mathrm{m}^{3}\right)$. $\mathrm{V}_{\leq \mathrm{i}}$ est le volume sortant par la vanne de vidange $\left(\mathrm{m}^{3}\right) . \mathrm{V}_{\mathrm{i}}$ représente les pertes par infiltration $\left(\mathrm{m}^{3}\right) . \mathrm{V}_{\mathrm{pr}}$ est le volume d'eau prélevé pour divers usages $\left(\mathrm{m}^{3}\right)$.

$$
\mathrm{Q}_{\mathrm{e}}=\mathrm{Q}_{\mathrm{d}}+\frac{\mathrm{dV}}{\mathrm{dt}}-\frac{\mathrm{dV}_{\mathrm{p}}}{\mathrm{dt}},
$$

où $\mathrm{Q}_{\mathrm{e}}$ est le débit instantané entrant dans le lac $\left(\mathrm{m}^{3} \mathrm{~s}^{-1}\right)$. $\mathrm{Q}_{\mathrm{d}}$ est le débit déversé $\left(\mathrm{m}^{3} \mathrm{~s}^{-1}\right)$. $\frac{\mathrm{dV}}{\mathrm{dt}}$ est la variation instantanée du volume d'eau retenue dans le lac $\left(\mathrm{m}^{3} \mathrm{~s}^{-1}\right) \cdot \frac{\mathrm{dV}_{\mathrm{p}}}{\mathrm{dt}}$ est le volume instantané directement précipité sur le lac obtenue à partir de la pluviographie $\left(\mathrm{m}^{3} \mathrm{~s}^{-1}\right)$.
Le débit de pointe est la valeur maximale de l'hydrogramme reconstitué $\mathrm{Q}_{\mathrm{e}}(t)$. Le volume de crue est déterminé par l'équation (6) (Onibon et al., 1997):

$$
\mathrm{V}_{\text {crue }}=\int \mathrm{Q}_{\mathrm{e}} \mathrm{dt} \approx \sum \frac{\mathrm{Q}_{\mathrm{e}}(\mathrm{t})-\mathrm{Q}_{\mathrm{e}}\left(\mathrm{t}_{\mathrm{i}-1}\right)}{2}\left(\mathrm{t}_{\mathrm{i}}-\mathrm{t}_{\mathrm{i}-1}\right),
$$

Pour ce travail, nous avons sélectionné 30 crues pour le bassin de Fidh Ali et 33 crues pour le cas du bassin Fidh Ben Naceur.

$\alpha$ et $\beta$ sont déterminé moyennant la méthode statistique d'optimisation (Onibon et al., 1997). Dans un premier temps, les produits $\alpha, \beta$ et K LS C P sont considérés constants, ayant la même valeur pour un bassin versant (Onibon et al., 1997). Par conséquent (eq. (1)) s'écrit sous la forme (eq. (7)) :

$$
\mathrm{A}_{\text {MUSLE }}=\lambda\left(\mathrm{q}_{\mathrm{p}} \mathrm{Q}\right)^{\beta}
$$

avec : $\lambda=\alpha \times \mathrm{K} \times \mathrm{LS} \times \mathrm{C} \times \mathrm{P}$.

Le paramètre $\beta$ est déterminé par la méthode des moindres carrées déjà appliquée à dix-huit bassins versants du réseau de la Dorsale tunisienne (Onibon et al., 1997 ; DCES/IRD, 1994 2002). Nous avons retenu pour ce coefficient $\beta$ la valeur de 0,623, qui est supérieure à celle donnée par Williams (1975) aux États-Unis, mais correspond aux travaux antérieurs dans cette zone de Tunisie (Onibon et al., 1997).

Le paramètre $\lambda$ est déterminé en comparant le transport solide calculé par l'équation (7) pour toutes les crues reconstituées entre deux mesures bathymétriques au transport solide calculé par l'équation (8) (Onibon et al., 1997). Selon Albergel (2008), l'équation (8) permet d'obtenir une bonne estimation des transports solides à l'exutoire d'un bassin versant aménagé avec une retenue. Elle globalise les pertes en terre dues aux trois formes d'érosion hydrique (Toy et al., 2002) : l'érosion en nappe qui provient du ruissellement des pluies sur les parcelles en pente des versants, le ravinement causé par les écoulements concentrés à l'échelle de portions de versants, et l'érosion des berges produite par les variations de régime d'écoulement dans le réseau hydrographique :

$$
A_{s}=\left(V_{o b s} \times d\right)+\sum_{j=1}^{n} S_{j} C_{j}
$$

où $A_{s}$ est le flux total des matières solides en suspension transporté entre deux campagnes de mesures bathymétriques (t). $V_{\text {obs }}$ est le volume de vase mesuré $\left(\mathrm{m}^{3}\right)$. d est la densité de la vase $\left(\mathrm{t} \mathrm{m}^{-3}\right)$. $\mathrm{n}$ est le nombre de crues ayant déversé entre deux mesures. $S_{j}$ est le volume d'eau déversé pendant la crue $\mathrm{j}\left(\mathrm{m}^{3}\right)$. $\mathrm{C}_{\mathrm{j}}$ est la concentration moyenne en matière solide mesurée pendant la crue $\mathrm{j}\left(\mathrm{t} \mathrm{m}^{-3}\right)$.

La densité de la vase varie selon le degré d'immersion des sédiments. Le volume de vase déposé entre deux dates est multiplié par une valeur moyenne de la densité $d=1,5 \mathrm{tm}^{-3}$ (Ben Mammou, 1998; DCES/IRD, 1994-2001; Jebari et al., 2010). Le volume d'eau déversé au-dessus du seuil pendant cette période est multiplié par une concentration moyenne en matière solide $C_{j}=0,015 \mathrm{t} \mathrm{m}^{-3}$ (Onibon et al., 1997).

Le paramètre $\alpha$ est obtenu une fois que $\lambda$ est connu.

Par conséquent, le facteur $\mathrm{R}_{\text {MUSLE }}$ est donné par les équations (9) et (10):

$$
\mathrm{R}_{\text {MUSLE, Fidh Ali }}=\sum_{\mathrm{j}=1}^{n} \frac{11,17}{238}\left(\mathrm{q}_{\mathrm{p}, \mathrm{j}} \mathrm{Q}_{\mathrm{j}}\right)^{0,623},
$$




$$
\mathrm{R}_{\text {MUSLE, Fidh Ben Naceur }}=\sum_{\mathrm{j}=1}^{n} \frac{8,38}{169}\left(\mathrm{q}_{\mathrm{p}, \mathrm{j}} \mathrm{Q}_{\mathrm{j}}\right)^{0,623}
$$

\subsubsection{Facteur d'érosivité de pluie $R_{\text {RUSLE }}$ pour le modèle RUSLE}

Le facteur $\mathrm{R}_{\mathrm{RUSLE}}$ est le facteur le plus important pour estimer la réponse de l'érosion au climat. Il dépend principalement de l'intensité pluviométrique et de l'énergie cinétique qui en résulte (Renard et al., 1997). Cette énergie, liée au diamètre des gouttes de pluie et à leur vitesse de chute, provoque le détachement des particules de sol et leur transport.

$\mathrm{R}_{\mathrm{RUSLE}}$ se calcule par le produit de l'énergie cinétique $\mathrm{E}$ (en mégajoules par hectare, $\mathrm{MJ} \mathrm{ha}^{-1}$ ) et de l'intensité maximale de la pluie $\left(I_{\max }\right)$; en Tunisie, Jebari (2009) a montré que l'I $\mathrm{I}_{\max }$ instantanée en $15 \mathrm{~min}$ (eq. (11)) est la plus appropriée pour quantifier les pertes en sol:

$$
\mathrm{R}_{\mathrm{RUSLE}}=\frac{\sum_{\mathrm{j}=1}^{j}\left(\mathrm{EI}_{15}\right)_{i}}{N},
$$

où $\left(\mathrm{EI}_{15}\right)_{\mathrm{i}}$ est la $(\mathrm{EI})_{15}$ pour un évènement $\mathrm{i}$, et $\mathrm{j}$ le nombre d'évènements pour $\mathrm{N}$ années dans une période d'étude.

Nous avons donc utilisé les données cumulées des précipitations toutes les $15 \mathrm{~min}$ sur la période 1994-2002 pour les stations Fidh Ali et Fidh Ben Naceur.

Les deux facteurs d'érosivité $R$, pour les deux modèles RUSLE et MUSLE, sont pris dans cette étude sous une forme unique et constante au sein de chaque bassin versant, du fait que leurs superficies sont assez faibles.

\subsubsection{Facteur d'érodibilité du sol K}

L'érodibilité du sol $\mathrm{K}$ caractérise la résistance d'un sol à l'érosion. Le facteur $\mathrm{K}$ est déterminé en fonction de caractéristiques des sols comme la texture, la présence de matière organique, la perméabilité et la profondeur. Dans la zone d'étude, on trouve trois catégories de sols : les marnes et argiles, les croûtes et les encroûtements calcaires, et les alluvions (Fig. 3a). Les valeurs de $\mathrm{K}$ pour chaque type sont tirées d'études antérieures.

\subsubsection{Facteur topographique (LS)}

L'indice LS combine la longueur de la pente (L) et son inclinaison (S). La longueur de la pente est la distance entre la limite supérieure de la parcelle et le point où l'eau de ruissellement commence à se concentrer au niveau d'un élément du réseau hydrographique. Les fortes pentes avec un écoulement rapide sont en général à l'origine d'une érosion importante. Le facteur LS est calculé pour chaque parcelle à partir du modèle numérique de terrain avec le logiciel System for Automated Geoscientific Analyses (Fig. 3b).

\subsubsection{Facteur de gestion de la couverture végétale (C)}

La couverture végétale du sol, sauf dans les endroits isolés, est sous l'influence directe des activités humaines, qui jouent donc fortement sur les pertes en sol. La couverture du sol influe sur la génération des processus d'infiltration de la pluie vers la

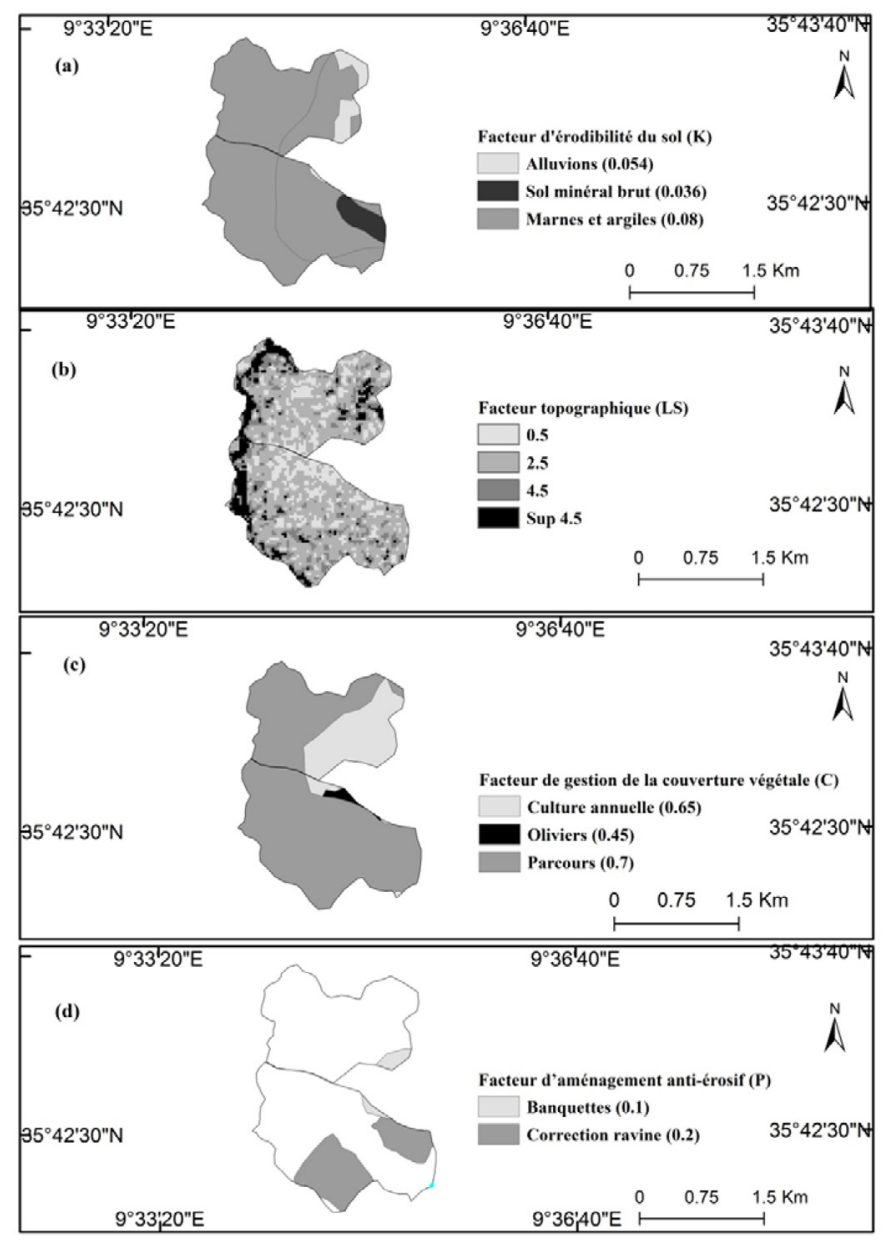

Fig. 3. Cartes des facteurs d'érosion utilisés dans les modèles RUSLE et MUSLE: (a) érodabilité du sol; (b) topographie; (c) couverture végétale; (d) aménagements anti-érosifs.

Fig. 3. Thematic map layers used in MUSLE and RUSLE models: (a) soil erodibility factor; (b) topographic factor; (c) crop management factor; (d) conservation practice factor.

zone non saturée et de ruissellement. Un même type de sol aura ainsi des comportements différents selon la couverture végétale. La zone d'étude se caractérise par trois types de couverture végétale: les parcours, les cultures annuelles, et quelques oliviers. Le facteur de gestion de la couverture végétale $C$ a été attribué pour chaque classe en utilisant les valeurs calculées dans des régions ayant des conditions agroclimatiques similaires (Fig. 3c).

\subsubsection{Facteur d'aménagement anti-érosif $(P)$}

Le facteur $P$ reflète les pratiques agricoles et de conservation du sol. Il réduit le ruissellement et la vitesse de l'eau, diminuant ainsi l'érosion hydrique. Les aménagements entrepris dans la zone étudiée sont constitués de plantations pastorales et arboricoles, de cordons et seuils en pierres sèches et de banquettes mécaniques. $\mathrm{P}=1$ pour les zones non aménagées et 0,1 pour la zone peu pentue aménagée avec des banquettes de rétention (Fig. 3d). 
Tableau 2a. Comparaison entre les mesures bathymétriques et les pertes en sol simulées par RUSLE-SIG et MUSLE-SIG. Table 2a. Comparison between bathymetric measurements and soil loss simulated by RUSLE-GIS and MUSLE-GIS.

\begin{tabular}{llll}
\hline Bassin versant & $\begin{array}{l}\text { Pertes en sol estimées } \\
\text { à partir des mesures } \\
\text { bathymétriques }\left(\mathrm{tha}^{-1} \mathrm{an}^{-1}\right)\end{array}$ & $\begin{array}{l}\text { Pertes en sol simulées par le } \\
\text { modèle RUSLE }\left(\mathrm{tha}^{-1} \mathrm{an}^{-1}\right)\end{array}$ & $\begin{array}{l}\text { Pertes en sol simulées par le } \\
\text { modèle MUSLE }\left(\mathrm{tha}^{-1} \mathrm{an}^{-1}\right)\end{array}$ \\
\hline Fidh Ali & 22,7 & 23,0 & 22,4 \\
Fidh Ban Naceur & 14,5 & 14,6 & 14,1 \\
\hline
\end{tabular}

Tableau 2b. Superficies par classes de pertes en sol simulées par RUSLE-SIG et MUSLE-SIG.

Table 2b. Soil loss classes areas simulated by RUSLE-GIS and MUSLE-GIS.

\begin{tabular}{|c|c|c|c|c|c|}
\hline \multirow[t]{2}{*}{ Bornes des classes $\left(\mathrm{tha}^{-1} \mathrm{an}^{-1}\right)$} & \multirow[t]{2}{*}{ Classe d'érosion } & \multicolumn{2}{|c|}{ RUSLE } & \multicolumn{2}{|c|}{ MUSLE } \\
\hline & & Superficie (ha) & Superficie $(\%)$ & Superficie (ha) & Superficie $(\%)$ \\
\hline $0-8$ & Moyenne & 168,4 & 41,4 & 177,1 & 43,5 \\
\hline$>20$ & Très forte & 120,4 & 29,6 & 116,6 & 28,6 \\
\hline
\end{tabular}

\subsection{Cartes d'estimation des pertes en sol}

Les cinq facteurs d'érosion ( $R, K, L S, C$ et $P$ ) sont intégrés dans un SIG avec le logiciel ArcGis sous forme de couches d'informations, avec la même résolution et le même système de coordonnées. Chaque couche est représentée par une image matricielle (raster). Les couches des différents facteurs sont ensuite superposées afin de fournir la distribution spatiale de l'érosion hydrique pour toutes les mailles de la zone.

Les deux approches MUSLE-SIG et RUSLE-SIG sont calibrées et validées afin de reproduire la réalité du terrain en termes de dégradations par l'érosion hydrique. Ces résultats sont comparés aux mesures bathymétriques au niveau des retenues collinaires Fidh Ali et Fidh Ben Naceur. Le passage des volumes des sédimentations $\left(\mathrm{m}^{3}\right)$ à des masses de pertes en sol ( $\left.\mathrm{tha}^{-1} \mathrm{an}^{-1}\right)$ se fait en utilisant la densité moyenne des sédiments (d) et l'équation (8). Le pourcentage d'erreur se calcule par l'équation (12):

$$
\% \text { erreur }=\frac{\mid \text { valeur simulée }- \text { valeur mesurée } \mid}{\text { valeur mesurée }} .
$$

Les simulations entreprises à l'échelle des deux bassins versants ont testé diverses tailles de maille (Jebari et al., 2012). Finalement, le choix de $25 \mathrm{~m} \times 25 \mathrm{~m}$ a été retenu puisqu'il permet de reproduire le plus convenablement les pertes en sol observées: des valeurs inférieures à $25 \mathrm{~m} \times 25 \mathrm{~m}$ sousestiment ici les pertes en sol et des valeurs supérieures à $25 \mathrm{~m} \times 25 \mathrm{~m}$ les surestiment.Pour des besoins de lisibilité de la carte, les valeurs d'érosion obtenues sont regroupées en classes.

\section{Résultats}

Les résultats fournis par les approches MUSLE-SIG et RUSLE-SIG permettent d'évaluer la répartition spatiale de l'érosion hydrique, d'identifier les zones vulnérables et de déterminer les pertes moyennes en sol, en $\mathrm{tha}^{-1} \mathrm{an}^{-1}$.
Le calcul de $\mathrm{R}$ entrepris considère respectivement le modèle MUSLE et RUSLE. Pour le bassin Fidh Ali, la valeur de R est égale à 276 et $485 \mathrm{MJ} \mathrm{mm} \mathrm{ha}{ }^{-1} \mathrm{hr}^{-1} \mathrm{an}^{-1}$ pour MUSLE et pour RUSLE. Pour le bassin versant Fidh Ben Naceur, elles sont égales à 334 et $303 \mathrm{MJ} \mathrm{mm} \mathrm{ha}{ }^{-1} \mathrm{hr}^{-1} \mathrm{an}^{-1}$, pour MUSLE et pour RUSLE.

Les pertes en sol moyennes annuelles correspondant au bassin versant Fidh Ali sont égales à 22,4 et 23,0 tha ${ }^{-1} \mathrm{an}^{-1}$, respectivement pour MUSLE et RUSLE (Tab. 2a). Le bassin versant Fidh Ben Naceur présente des pertes annuelles de 14,1 $\mathrm{tha}^{-1} \mathrm{an}^{-1}$ (MUSLE) et 14,6 $\mathrm{h} \mathrm{ha}^{-1} \mathrm{an}^{-1}$ (RUSLE) (Tab. 2a).

Les pertes en sol simulées par les deux modèles sont proches des valeurs mesurées (Tab. 2a), avec une erreur faible, de l'ordre de $2 \%$ pour MUSLE et $1 \%$ pour RUSLE. L'écart entre les érosions hydriques simulées par MUSLE et RUSLE est faible (Tab. 2a); les deux modèles sont donc aussi satisfaisants l'un que l'autre.

\section{Discussion}

La distribution spatiale des pertes en sol simulée par les approches MUSLE-SIG et RUSLE-SIG montre une hétérogénéité de la zone d'étude (Fig. 4). En effet, une érosion moyenne inférieure à $8 \mathrm{tha}^{-1} \mathrm{an}^{-1}$ est simulée sur presque la moitié de la zone étudiée : $43,5 \%$ pour MUSLE-SIG et $41,4 \%$ pour RUSLE-SIG. Les surfaces avec une faible érosion hydrique se situent principalement dans la partie nord-est de la zone. Les endroits à très forte érosion hydrique, supérieure à $20 \mathrm{tha}^{-1} \mathrm{an}^{-1}$, occupent environ $30 \%$ de la superficie et se concentrent dans la partie nord-ouest (Tab. 2b). Cela est dû à plusieurs facteurs, comme le substratum géologique dominé par des roches très imperméables, les fortes pentes et les activités humaines (intensification agricole et élevage). Dans cette zone, les sols se sont formés sur des matériaux tendres tels que marnes et gypses, peu perméables, ce qui favorise le ruissellement et l'érosion (Bouzaiane et Laforgue, 1986). Ceci a engendré des bad-lands (paysage ruiniforme raviné par le 

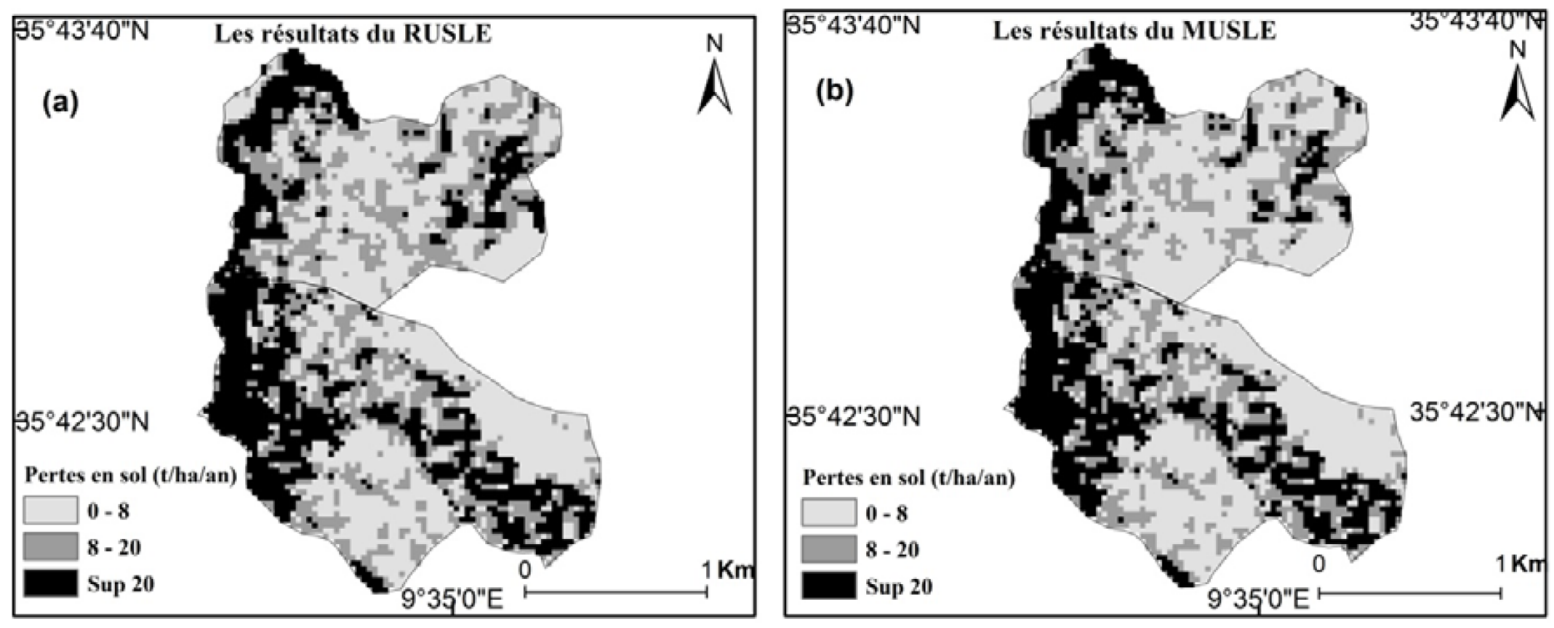

Fig. 4. Répartition spatiale de l'érosion hydrique (a) avec RUSLE (b) avec MUSLE.

Fig. 4. Spatial distribution of soil erosion (a) with RUSLE (b) with MUSLE.

(a)

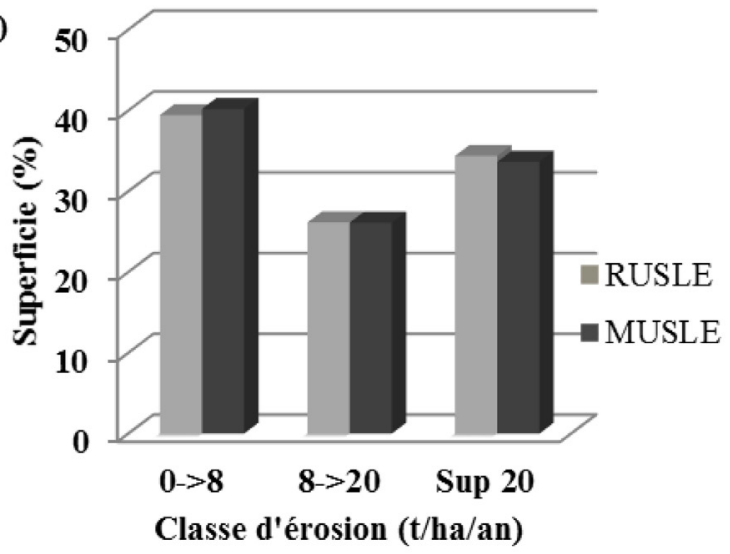

(b)

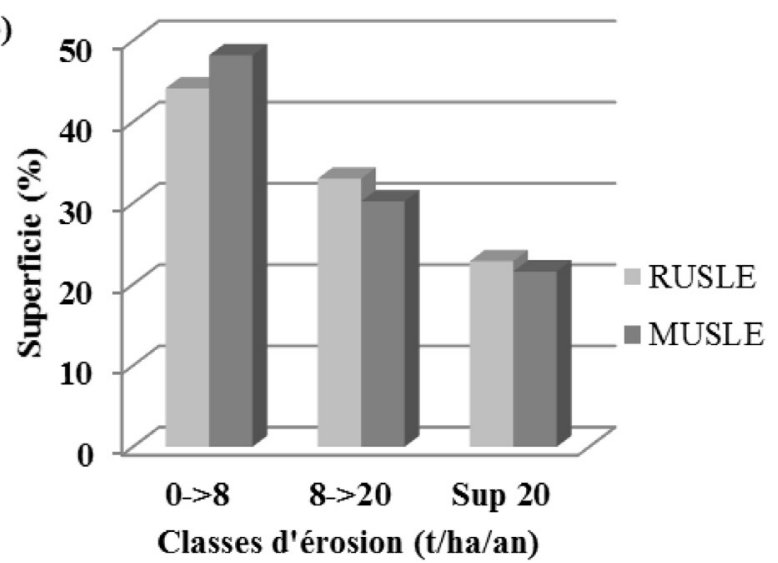

Fig. 5. Classes de pertes en sol simulées par RUSLE et MUSLE pour: (a) Fidh Ali; (b) Fidh Ben Naceur.

Fig. 5. Soil loss classes using RUSLE and MUSLE models for: (a) Fidh Ali; (b) Fidh Ben Naceur.

ruissellement) dans certaines parties de la zone. Les sédiments détachés du haut du versant sont susceptibles de se déposer en bas de pente plutôt que d'être emportés hors du bassin (Mounirou, 2012), avec donc une sédimentation locale. Par ailleurs, les dépôts dans les axes d'écoulement et les dépressions superficielles contribuent au comblement des cours d'eau et entraînent des difficultés de drainage (Mounirou, 2012). Ce contexte favorise l'instabilité des pentes, les glissements de terrain et le ravinement (Foucault et al., 2014).

L'action anthropique contribue à accélérer l'érosion hydrique. Sont en cause le choix des systèmes culturaux et l'exploitation intensive des terres avec des pratiques agricoles inadaptées: labour trop fréquent et dans le sens de la pente, mauvaise maîtrise de l'irrigation et de l'épandage, surpâturage (Braiki, 2018).

Les pertes en sol simulées par les deux modèles MUSLESIG et RUSLE-SIG sont similaires. Ceci est vrai aussi bien pour les valeurs moyennes annuelles que pour la répartition spatiale. Les différences entre les deux modèles sont insignifiantes et non perceptibles au niveau de la cartographie (Fig. 5).
Avec un paramétrage adéquat, MUSLE-SIG et RUSLESIG sont donc tous deux bien adaptés à la zone semi-aride tunisienne. MUSLE-SIG serait ainsi très utile pour estimer l'érosion hydrique et sa distribution à partir des données hydrométriques (débit et volume du ruissellement), là où les hauteurs de pluie ne sont pas disponibles à de faibles pas de temps (15 ou $30 \mathrm{~min}$ ), comme le demande le modèle RUSLE.

Bien qu'il existe dans la littérature des travaux qui relient le coefficient SDR (Sediment Delivery Ratio) au modèle RUSLE (Lim et al., 2005 ; Yoon et al., 2009; Gelagay, 2016), le présent travail n'a pas mobilisé le SDR. En effet, plusieurs publications montrent que le SDR présente de fortes incertitudes (Klaghofer et al., 1992 ; Benavidez et al., 2018).

Cette étude montre que la modélisation des pertes en sol et de leur distribution spatiale aboutit à des résultats conformes à la réalité du terrain. Les approches considérées ont donc une fiabilité satisfaisante pour décrire la dégradation du paysage agricole par l'érosion hydrique. Ces résultats consolident les travaux expérimentaux et théoriques conduits de longue date (plus de 60 ans) en Tunisie et permettent de valoriser des 
acquis scientifiques issus de collaborations internationales multidisciplinaires.

Ce travail reste encore pionnier dans la région MENA (Middle East and North Africa). L'approche adoptée étant prometteuse, il serait désormais possible de l'étendre à d'autres sites d'étude, et de considérer des échelles plus étendues.

\section{Conclusion}

Ce travail a permis de quantifier les pertes en sol en utilisant l'Equation universelle des pertes en sol (USLE) dans ses versions Modifiée (MUSLE) et Révisée (RUSLE), couplées à un SIG. L'approche utilisée a permis de calibrer et valider les deux modèles avec des observations de terrain. Nous avons ainsi valorisé les données hydrologiques, cartographiques et bathymétriques disponibles. La démarche a permis de quantifier les pertes en sol annuelles ainsi que d'identifier les zones les plus vulnérables dans les deux bassins versants étudiés. Les résultats montrent une reproduction fidèle des valeurs de l'érosion ainsi que de la dégradation du paysage agricole. La comparaison des pertes en sol simulées avec celles mesurées montre un écart faible, de l'ordre de 1 à $2 \%$. Par conséquent, cette démarche de simulation pourrait être utilisée par les services techniques agricoles centraux et régionaux là où les observations hydrologiques font défaut. La modélisation peut ainsi permettre aux praticiens de l'aménagement rural et du développement agricole de mesurer plus rapidement et à faible coût la vulnérabilité du paysage agricole, et donc de proposer des solutions plus adaptées pour conserver les ressources en eau et en sol dans des zones semi-arides, préserver les infrastructures hydrauliques et faciliter ainsi le développement rural et une activité agricole moins génératrice d'érosion.

Les résultats simulés par chacun des deux modèles, MUSLE-SIG et RUSLE-SIG, sont pratiquement identiques. L'usage de l'un ou de l'autre est conditionné par la nature des données disponibles. En effet, si MUSLE exige des données sur le débit de pointe et le volume du ruissellement, RUSLE nécessite une mesure pluviométrique à faible pas de temps. Les informations nécessaires sur la couverture du sol, la topographie et la nature du sol, sont les mêmes dans les deux cas.

Les approches MUSLE-SIG et RUSLE-SIG utilisées ici ont mobilisé les versions les plus récentes, améliorées et validées par la communauté scientifique. Malgré les limites de ces modèles mentionnées dans la littérature, certaines innovations permettent de les surmonter. Ainsi, le couplage avec un SIG permet le maillage et le découpage en parcelles. Ceci rend possible l'estimation de la distribution spatiale de l'érosion hydrique dans des zones vastes (bassin versant, pays ou continent). La prise en compte du contexte climatique local permet d'adopter une valeur d'érosivité adaptée aux conditions semi-arides. Les observations sédimentologiques et érosives permettent le calage et la validation du modèle dans un contexte hydrologique particulier.

Le modèle RUSLE est actuellement le plus utilisé en Tunisie, avec des résultats satisfaisants. Il devrait rester le plus prisé, y compris par le secteur du développement, puisque le réseau tunisien d'observation pluviométrique est plus dense que le réseau hydrométrique. Le modèle MUSLE reste une option intéressante quand les données pluviométriques font défaut et que des données hydrométriques sont disponibles. Il reste cependant à promouvoir un transfert plus important de ces approches au monde du développement agricole, pour des applications à des zones plus larges.

Ce travail montre aussi l'importance de la continuité des recherches, entamées en Tunisie depuis les années 1950. C'est cette continuité qui a permis d'acquérir et d'affiner les paramètres nécessaires au fonctionnement des modèles MUSLE et RUSLE. C'est le cumul de tous les efforts consentis pendant plus de 60 ans qui permet aujourd'hui de quantifier et localiser convenablement les pertes en sol, de simuler l'impact de l'anthropisation, et donc de guider les agents du développement rural pour définir là où les aménagements hydro-agricoles et anti-érosifs devraient être installés en priorité, mais aussi pour étudier les conséquences du changement climatique sur les paysages agricoles.

Remerciements. Les auteurs tiennent à remercier pour leur appui le projet FASTER, grant $\mathrm{n}^{\mathrm{o}} 810812$, financé par l'Union européenne dans le cadre du programme H 2020, et le programme PACTE mis en œuvre par la DG/ACTA et financé par l'AFD et le FFEM.

\section{Références}

Albergel J, Nasri S, Lamachère JM. 2004. Hydromed : programme de recherche sur les lacs collinaires dans les zones semi-arides du pourtour méditerranéen. Revue des sciences de l'eau 17(2): 133 151. https://doi.org/10.7202/705526ar.

Albergel J. 2008. Place des petits barrages dans la mobilisation des eaux de surface et dans la lutte contre l'érosion au Maghreb et au Moyen-Orient. In: Roose E, Albergel J, De Noni G, Sabir M, Laouina A, eds. Efficacité de la GCES en milieu semi-aride. Paris (France): AUF, EAC et IRD éditeurs, pp. 35-45.

Alewell C, Borrelli P, Meusburger K, Panagos P. 2019. Using the USLE: chances, challenges and limitations of soil erosion modelling. International Soil and Water conservation Research 7(3): 203-225. https://doi.org/10.1016/j.iswcr.2019.05.004.

Benavidez R, Jackson B, Maxwell D, Norton K. 2018. A review of the (Revised) Universal Soil Loss Equation ((R)USLE): with a view to increasing its global applicability and improving soil loss estimates. Hydrology and Earth System Sciences 22(11): 6059 6086. https://doi.org/10.5194/hess-22-6059-2018.

Ben Mammou A. 1998. Barrages Nebeur, Sidi Salem, Sidi Saad, et Sidi Bou Baker. Quantification, étude sédimentologique et géotechnique des sédiments piégés. Apport des images satellitaires. Thèse de doctorat. Sciences géologiques. Tunisie: Université de Tunis II, Faculté des Sciences de Tunis.

Borrelli P, Robinson DA, Fleischer LR, Lugato E, Ballabio C, Alewell C, et al. 2017. An assessment of the global impact of 21 st century land use change on soil erosion. Nature Communications 8: 1-13. https://doi.org/10.1038/s41467-017-02142-7.

Boufaroua M, Albergel J, Pépin Y. 2000. Bilan de l'érosion sur les petits bassins versants des lacs collinaires de la dorsale Tunisienne. In: Annuaire des publications de l'association internationale des géologues, pp. 1209-1216.

Bouzaiane S, Laforgue A. 1986. Monographie hydrologique des oueds Zéroud et Merguellil. Tunis (Tunisie), Paris (France): ministère de l'Agriculture - ORSTOM, $1058 \mathrm{p}$.

Braiki H. 2018. Construction d'une démarche participative pour améliorer la gestion de l'eau et du sol, Une application aux 
politiques des aménagements de conservation des eaux et des sols en Tunisie Centrale. Thèse de doctorat. Sciences de l'environnement. Montpellier: AgroParisTech, Institut National Agronomique de Tunisie, $220 \mathrm{p}$.

Camus H, Guiguen N, Ben Younes M. 1995. Note sur l'envasement des lacs collinaires en zone semi-aride tunisienne. Direction de la conservation des eaux et du sol et l'Institut de recherche pour le développement en coopération (ORSTOM). Rapport, 49 p.

Collinet J, Zante P, Attia R, Dridi B, Agrebaoui S. 2002. Analyse expérimentale de l'érosion aréolaire sur le bassin versant du lac collinaire de Fidh Ali (Tunisie). Direction des sols et l'Institut de recherche pour le développement mission IRD de Tunis. Rapport, $49 \mathrm{p}$.

Cinnirella S, Iovino F, Porto P, Ferro V. 1998. Anti-erosive effectiveness of Eucalyptus coppices through the cover management factor estimate.Hydrological Processes 12(4): 635-649. https://doi.org/10.100/(SICI)1099-1085(19980330)12:4\%3C635:: AID-HYP598\%3E3.0.CO;2-Q.

CNEA. 2008. Étude stratégique sur le développement durable et l'agriculture dans les gouvernorats de Gabes, Sidi Bouzid, Nabeul, le Kef et Bizerte. Tunis: ministère de l'Agriculture, Centre national des études agricoles.

CREGR. 1965. Utilisation pratique de l'équation de perte de sol. Fiche technique de conservation des eaux et du sol (26/2/1). Tunis (Tunisie): CREGR.

DCES/IRD. 1994-2001. Annuaires hydrologiques des lacs collinaires. Rapports 1994-2001. Tunis: Direction générale de l'aménagement et de la conservation des terres agricoles et Institut de recherche pour le développement.

El Amami S. 1984. Les aménagements hydrauliques traditionnels de Tunisie. Tunis: ministère de l'Agriculture, Centre de recherche du génie rural.

Foucault A, Raoult JF, Cecca F, Platevoet B. 2014. Dictionnaire de géologie, $8^{\mathrm{e}}$ éd. Paris (France): Dunod, $416 \mathrm{p}$.

Gao J, Wang H. 2018. Temporal analysis on quantitative attribution of karst soil erosion: a case study of a peak-cluster depression basin in Southwest China. Catena (172): 369-377. https://doi.org/10.1016/ j.catena.2018.08.035.

Gelagay HS. 2016. RUSLE and SDR Model Based Sediment Yield Assessment in a GIS and Remote Sensing Environment; a case study of Koga Watershed, Upper Blue Nile Basin, Ethiopia. Hydrology Current Research 7: 239. https://doi.org/10.4172/ 2157-7587.1000239.

Guy H. 1967. Synthèse des notes rédigées en matière de conservation des eaux et du sol au centre de Génie rural. P.E.N. AGIR3. S1. Tunis Tunisie: CREGR.

Heush B. 1982. Étude de l'érosion et des transports solides en zone semi-aride. Recherche bibliographique sur l'Afrique du Nord. PNUD, Projet Rab/80/ 04.

Jebari S, Berndtsson R, Bahri A, Boufaroua M. 2008. Exceptional rainfall characteristics related to erosion risk in semi-arid Tunisia. The Open Hydrology Journal 2(1): 25-33. DOI: 10.2174/ 1874378100802010025 .

Jebari S. 2009. Water erosion modeling using fractal rainfall disaggregation - A study in semiarid Tunisia. PhD Thesis, Rep. 1047, Department of Water Resource Engineering. Sweden: Lund University, $140 \mathrm{p}$.

Jebari S, Berndtsson B, Bahri A, Boufaroua M. 2010. Spatial soil loss risk and reservoir siltation in semi-arid Tunisia. Hydrological Sciences Journal 55(1): 121-137. https://doi.org/10.1080/ 02626660903529049.

Jebari S, Berndtsson B, Olsson J, Bahri A. 2012. Soil erosion estimation based on rainfall disaggregation. Journal of Hydrology
(436-437): 102-110. https://doi.org/10.1016/j.jhy drol.2012.03.001.

Jebari S, Berndtsson R, Bahri A. 2015. Challenges of traditional rainwater harvesting systems in Tunisia. Middle East Critique 24 (3): 289-306. https://doi.org/10.1080/19436149.2015.1046707.

Klaghofer E, Summer W, Villeneuve JP. 1992. Some remarks on the determination of the sediment delivery ratio. Erosion, Debris Flows and Environment in Mountain Regions, Proceedings of the Chengdu Symposium, July 1992. IAHS Publ. 209: 113-118.

Ketema A, Dwarakish GS. 2019. Water erosion assessment methods: a review. ISH Journal of Hydraulic Engineering 1-8. https://doi. org/10.1080/09715010.2019.1567398.

Kinnell PIA. 2010. Event soil loss, runoff and the universal soil loss equation family of models: a review. Journal of Hydrology 385(14): 384-397. https://doi.org/10.1016/j.jhydrol.2010.01.024.

Kirkby MJ, Jones RJA, Irvine B, Gobin A, Govers G, Cerdan O, et al. 2003. Pan European soil erosion risk assessment: the PESERA map, version 1. esdac.jrc.ec.europa.eu/public_path/Pesera.pdf.

Le Bissonnais Y, Montier C, Jamagne M, Daroussin J, King D. 2002. Mapping erosion risk for cultivated soil in France. Catena 46(2-3): 207-220. https://doi.org/10.1016/S0341-8162(01)00167-9.

Lim KJ, Sagong M, Engel BA, Tang Z, Choi J, Kim KS. 2005. GISbased sediment assessment tool. Catena 64(1): 61-80. https://doi. org/10.1016/j.catena.2005.06.013.

Masson JM. 1970. L'érosion des sols par l'eau en climat méditerranéen : méthodes expérimentales pour l'étude des quantités érodées à l'échelle du champ. Thèse de doctorat. Montpellier (France), $213 \mathrm{p}$.

Masson JM. 1972. L'érosion des sols par l'eau en climat méditerranéen. Méthodes expérimentales pour l'étude des quantités érodées à l'échelle du champ. La Houille Blanche (8): 673 678. https://doi.org/10.1051/lhb/1972048.

Mhiri A, Ben Said M, Kaabia M, Bachta MS. 1994. Pour une approche holistique de la restauration des terres soumises à l'érosion hydrique dans le nord-ouest de la Tunisie. In: Congrès international sur la restauration et la réhabilitation des terres dégradées en zones arides et semi-arides. 14-16 novembre, Djerba (Tunisie).

Mounirou L. 2012. Étude du ruissellement et de l'érosion à différentes échelles spatiales sur le bassin versant de Tougou en zone sahélienne du Burkina Faso : quantification et transposition des données. Thèse de Doctorat. France: Université de Montpellier.

Nasri S. 2002. Hydrological effects of water harvesting techniques: a study of Tabias, soil contour ridges, and hill reservoirs in Tunisia. PhD Thesis, Rep. 1030, Department of Water Resource Engineering. Sweden: Lund University, $104 \mathrm{p}$.

Onibon H, Albergel J, Bargaoui Z, Pepin Y. 1997. Modélisation des transports solides sur les bassins versants des lacs collinaires. In: Journées scientifiques de l'INRGREF, Monastir (Tunisie).

PNUD/FAO. 1991. TUN. 86-020-Rapport d'évaluation sur les techniques de CES en Tunisie. Tunis: Direction CES, ministère de l'Agriculture.

Renard KG, Foster GR, Weesies GA, McCool DK. 1997. Predicting soil erosion by water - a guide to conservation planning with the revised universal soil loss equation (RUSLE). Washington, USDA. Agriculture Handbook 703: 404 p.

Renard KG, Yoder DC, Lightle DT, Dabney SM. 2011. Universal soil loss equation/revised universal soil loss equation. In: Morgan RPC, Nearing MA, eds. Handbook of erosion modeling. Oxford (England): Blackwell Publishing Ltd., pp. 137-167. https://doi. org/10.1002/9781444328455.ch8.

Saadaoui M. 1996. Modélisation des évènements averses-cruestransport solide: cas du barrage collinaire d'El Ogla. In: $18^{\mathrm{e}}$ Journée 
des ressources en eau. Le transport solide des oueds en Tunisie. Tunis (Tunisie), INAT, 26 avril 2001.

Smith HJ, Van Zyl AJ, Claassens AS, Schoeman JL, Laker MC. 2000. Soil loss modeling in the Lesotho Highlands water project catchment areas. South African Geographical Journal 82(2): 6469. https://doi.org/10.1080/03736245.2000.9713694.

Sun W, Shao Q, Liu J, Zhai J. 2014. Assessing the effects of land use and topography on soil erosion on the Loess Plateau in China. Catena 121: 151-163. https://doi.org/10.1016/j. catena.2014.05.009.

Toy JT, Foster GR, Renard KG. 2002. Soil erosion: processes, prediction, measurement and control. New York ( USA): Wiley, 352 p.

Williams JR. 1975. Sediment-yield prediction with universal equation using runoff energy factor. In: Present and prospective technology for predicting sediment yield and sources. Washington (USA): ARS.S-40, US Gov. Print. Office, pp. 244-252.

Wischmeier WH, Smith D. 1965. Prediction rainfall erosion losses from cropland east of the Rocky Mountains: a guide for selection of practices for soil and water conservation. Washington, USDA. Agricultural Handbook 282: 47 p.

Yoon KS, Kim C, Woo H. 2009. Application of RUSLE for erosion estimation of construction sites in coastal catchments. Journal of Coastal Research: 1696-1700.

Yue Y, Ni JR, Ciais P, Piao SL, Wang T, Huang MT, et al. 2016. Lateral transport of soil carbon and land-atmosphere $\mathrm{CO} 2$ flux induced by water erosion in China. Proceedings of the National Academy of Sciences of the United States of America 113(24): 6617-6622. https://doi.org/10.1073/pnas.1523358113.

Citation de l'article : Khemiri K, Jebari S. 2021. Évaluation de l'érosion hydrique dans des bassins versants de la zone semi-aride tunisienne avec les modèles RUSLE et MUSLE couplés à un Système d'information géographique. Cah. Agric. 30: 7. 